\title{
ZERO-DIMENSIONAL SCHEMES IN THE PLANE
}

\author{
E. BALLICO
}

\begin{abstract}
Let $Z \subset \mathbb{P}^{2}$ be a zero-dimensional scheme. Fix $t \in \mathbb{N}$. In this paper we study the following question: find assumptions on $Z$ and $t$ such that $h^{1}\left(\mathcal{I}_{A}(t)\right)<h^{1}\left(\mathcal{I}_{Z}(t)\right)$ for all $A \subsetneq Z$ and check if $t$ does not exist for a certain class of schemes $Z$.
\end{abstract}

\section{INTRODUCTION}

In the last few years at least two areas of applied mathematics saw a surge of interest in the following abstract topic: the cohomology groups $H^{1}\left(\mathcal{I}_{Z}(t)\right)$, $t \in \mathbb{Z}$, where $Z \subset \mathbb{P}^{r}$ is a zero-dimensional scheme.

One topic is related to symmetric tensors $([16])$. A cursory look at the abstracts of [3] and [4] shows that there is a huge difference between the case $r=2$ and the case $r>2$. Often the proof for arbitrary $r$ is done by induction on $r$ and in the case $r=2$ one may use (as in [3]) the more elementary consequences of [12]. Motivated by the proofs in the quoted papers we looked at a similar abstract problem $([2])$.

The other topic is coding theory. Here the minimum distance and the small (but not minimal) weights of certain evaluation codes are obtained looking at $Z$ and $t$ with $h^{1}\left(\mathcal{I}_{Z}(t)\right)>0$ (or with $h^{1}\left(\mathcal{I}_{Z}(t)\right)=e$ for some $e \geq 2$ if we look at the higher weights of the dual code as in ([13], [1]). Again, a cursory look at the papers which quote [9] show that in the case of plane curves (e.g. the Hermitian curve) the results are far stronger. For plane curves the authors used [12] in [6], [7], [8], [1]. In our opinion the paper [12] is a key tool which makes the difference between the case $r=2$ and the case $r>2$. This paper is devoted to the spelling out of this opinion (see section 4 for several stumbling blocks to make this opinion a fact).

Let $Z \subset \mathbb{P}^{2}$ be a zero-dimensional scheme. Assume $Z \neq \emptyset$. Let $s(Z)$ be the minimal integer $t$ such that $h^{0}\left(\mathcal{I}_{Z}(t)\right)>0$. Let $\tau(Z)$ be the maximal integer $t$ such that $h^{1}\left(\mathcal{I}_{Z}(t)\right)>0$. Let $h_{Z}$ be the Hilbert function of $Z$,

2010 Mathematics Subject Classification. 14N05.

Key words and phrases. Zero-dimensional scheme, plane curve, Hilbert function.

The author was partially supported by MIUR and GNSAGA of INdAM (Italy). 
i.e. set $h_{Z}(t):=\operatorname{deg}(Z)-h^{1}\left(\mathcal{I}_{Z}(t)\right)$ for all $t \in \mathbb{N}$. The knowledge of the Hilbert function $h_{Z}(t), t \in \mathbb{N}$, is equivalent to the knowledge of the numerical character $n_{0}, \ldots, n_{s-1}$ of $Z$ ([15], [12]). We recall that $s=s(Z), n_{i} \in \mathbb{N}$ for all $i, n_{0} \geq \cdots \geq n_{s-1} \geq s$ and

$$
h^{1}\left(\mathcal{I}_{Z}(t)\right)=\sum_{i=0}^{s-1}\left(\max \left\{0, n_{i}-t-1\right\}-\max \{0, i-t-1\}\right)
$$

for each $t \in \mathbb{Z}$. Hence $\tau(Z)=n_{0}-2$ and $\operatorname{deg}(Z)=\sum_{i=0}^{s-1}\left(n_{i}-i\right)$ (take $t=-1$ in (1)). We fix positive integers $t, e$ and look at all schemes $Z$ such that $h^{1}\left(\mathcal{I}_{Z}(t)\right)=e$ and $h^{1}\left(\mathcal{I}_{A}(t)\right)<e$ for all $A \subsetneq Z$. Let $s, \tau, n_{i}$ be the invariants of such a scheme $Z$. Since $e>0$, we have $t \leq \tau$. Since $\tau=n_{0}-2$ and $n_{0} \geq \cdots \geq n_{s-1} \geq s$, we have $\tau \geq s-2$. The cases $\tau=s-2$ and $\tau=s-1$ are very easy (Proposition 2). We recall that the sequence $n_{0} \geq \cdots \geq n_{s-1}$ is said to be connected if $n_{t} \geq n_{t-1}-1$ for all $t \in\{1, \ldots, s-1\}$. If $n_{0}, \ldots, n_{s-1}$ is not connected, any $t \in\{1, \ldots, s-1\}$ with $n_{t} \leq n_{t-1}-2$ is called a gap.

The interesting part of this paper arises when the numerical character of $Z$ has gaps and the only part not contained in [12] (explicitly or implicitly) is when there are at least two gaps. For any zero-dimensional scheme $W \subset \mathbb{P}^{2}$ and any effective divisor $T \subset \mathbb{P}^{2}$ let $\operatorname{Res}_{T}(W)$ denote the zero-dimensional subscheme of $\mathbb{P}^{2}$ with $\mathcal{I}_{W}: \mathcal{I}_{T}$ as its ideal sheaf. We have $\operatorname{deg}(W)=\operatorname{deg}(W \cap$ $T)+\operatorname{deg}\left(\operatorname{Res}_{T}(W)\right)$. Assume that the numerical character $n_{0} \geq \cdots \geq n_{s-1} \geq$ $s$ of $Z$ has $c>0$ gaps. Let $k_{1}, \ldots, k_{c}, c \geq 1$, be the gaps of $n_{0}, \ldots, n_{s-1}$. There are uniquely determined curves $T_{i} \subset \mathbb{P}^{2}, 1 \leq i \leq c$, with the following property. Set $Z_{0}:=Z$. Let $T_{1}$ be be the only degree $k_{1}$ curve such that $E_{1}:=Z \cap T_{1}$ has numerical character $n_{0}, \ldots, n_{k_{1}-1}$ and $Z_{1}:=\operatorname{Res}_{T_{1}}(Z)$ has numerical character $m_{0}, \ldots, m_{s-k_{1}-1}$, where $m_{i}:=n_{i}-k_{1}$ for all $i$ (Lemma 8 ). If $c \geq 2$, then the numerical character of $Z_{1}$ is not connected and $k_{2}-k_{1}$ is its first non-gap. Let $T_{2} \subset \mathbb{P}^{2}$ be the unique degree $k_{2}-k_{1}$ curve such that $E_{2}:=Z_{1} \cap T_{1}$ has numerical character $m_{0}, \ldots, m_{k_{2}-1}$ and $Z_{2}:=\operatorname{Res}_{T_{2}}\left(Z_{1}\right)$ has numerical character $a_{0}, \ldots, a_{s-k_{2}-1}$ with $a_{i}:=n_{i}-k_{2}$ for all $i$ (Lemma 8). If $c \geq 3$, then we define recursively the degree $k_{i+1}-k_{i}$ curve $T_{i+1}$ and the zero-dimensional schemes $E_{i+1}:=T_{i+1} \cap Z_{i}$ and $\operatorname{Res}_{T_{i+1}}\left(Z_{i}\right)$ quoting [12]. We call the sequences $T_{1}, \ldots, T_{c}, E_{1}, \ldots, E_{c}, E_{c+1}, Z_{1}, \ldots, Z_{c}=E_{c+1}$ (or, sometimes, just $E_{1}, \ldots, E_{c}, E_{c+1}$ ) the splitting sequences of $Z$. Notice that each scheme $E_{i}$ has a connected numerical character. The scheme $E_{i}$ uniquely determines $T_{i}$ (Remark 4 ). We have $E_{c+1}=Z_{c}$, but often the sequence $E_{1}, \ldots, E_{c+1}$ does not determine $Z$, even if each $E_{i}$ is reduced (Example 3). Of course, if $Z$ is reduced, then the sequence $E_{1}, \ldots, E_{c+1}$ determines $Z$, because $Z=E_{1} \sqcup \cdots \sqcup E_{c+1}$. In summary, when the numerical character of $Z$ has $c \geq 1$ gaps we associate to $Z$ the following data which are uniquely determined by $Z$ : 
- $c$ effective divisors $T_{1}, \ldots, T_{c} \subset \mathbb{P}^{2}$;

- A filtration of subschemes of $Z_{0}:=Z \supset Z_{1} \supset \cdots \supset Z_{c}$ with $Z_{i}=$ $\operatorname{Res}_{T_{i}}\left(Z_{i-1}\right), 1 \leq i \leq c$.

- The schemes $E_{i}:=T_{i} \cap Z_{i-1}, 1 \leq i \leq c$, and the scheme $E_{c+1}:=Z_{c}$ with prescribed numerical character; the numerical character of each $E_{i}$ has no gaps; each $E_{i}, 1 \leq i \leq c$, uniquely determines the curve $T_{i}$ (Remark 4).

For instance if $Z$ is reduced and the numerical character has $c$ gaps, then we get a decomposition $Z=E_{1} \sqcup \cdots \sqcup E_{c+1}$ in which each set $E_{i}$ is uniquely determined and each $E_{i}, 1 \leq i \leq c$, determines $T_{i}$ (see section 3 for the details). Most papers before [12] were concerned in cases in which essentially there was one potential gap and the goal was to give sharp conditions assuring that $Z$ is a complete intersection or that it has a certain CayleyBacharach property ([10]). Apparently, none of these results are related to our problem (we are trying to find if there is a proper $Z_{e} \subsetneq Z$ which is extremal for the Hilbert function in certain ranges). Any admissible Hilbert function (i.e. any numerical character) is realized by some zero-dimensional scheme and we may even take a reduced scheme $([10], \S 4)$. Hence obviously among the examples in [10], §4, there are examples of sets $E_{1} \sqcup \cdots \sqcup E_{c+1}$. But this is not very useful in the applications, say to codes associated to a smooth plane curve. Let $C \subset \mathbb{P}^{2}$ be a smooth plane curve defined over $\mathbb{F}_{q}$ and suppose you have a Goppa code $\mathcal{C}$ on $C$ associated with the embed$\operatorname{ding} C \hookrightarrow \mathbb{P}^{2}$, e.g., the dual of the code obtained evaluating all linear forms of degree $d$ at a certain subset of $C\left(\mathbb{F}_{q}\right)([6],[7],[8])$. Many properties of this code (e.g. minimum distance, the enumeration of all codewords with small weight) may be translated into the existence or not of zero-dimensional schemes $Z \subset C$ for which we prescribe the degree and the first part of its numerical character (see section 4). If from these informations we get the existence of at least one gap, then we are in good shape, because we may often construct examples taking $T_{1}, \ldots, T_{e}$ and $Z \subset C \cap\left(T_{1}+\cdots+T_{e}\right.$ ) (with care if $T_{i}$ and $T_{j}$ have a common components for some $\left.i \neq j\right)$.

Fix a zero-dimensional scheme $Z \subset \mathbb{P}^{2}$ and an integer $t>0$ such that $\gamma:=h^{1}\left(\mathcal{I}_{Z}(t)\right)>0$. Notice that $h^{1}\left(\mathcal{I}_{A}(t)\right) \geq \gamma+\operatorname{deg}(A)-\operatorname{deg}(Z)$ for every scheme $A \subset Z$. We say that $Z$ is strongly defective in degree $t$ if for all schemes $A \subset Z$ we have $h^{1}\left(\mathcal{I}_{A}(t)\right)=\max \{0, \gamma+\operatorname{deg}(A)-\operatorname{deg}(Z)\}$.

A zero-dimensional scheme $Z \subset \mathbb{P}^{2}$ has maximal rank if for each $t \in \mathbb{Z}$ either $h^{1}\left(\mathcal{I}_{Z}(t)\right)=0$ or $h^{0}\left(\mathcal{I}_{Z}(t)\right)=0$. Hence $Z$ has maximal rank if and only if $\tau(Z)<s(Z)$.

A zero-dimensional scheme $Z \subset \mathbb{P}^{2}$ is said to be in uniform position in degree $t$ or to have uniform position in degree $t$ if for every degree $t$ curve $T \subset \mathbb{P}^{2}$ either $Z \subset T$ or $\operatorname{deg}(Z \cap T)<\left(\begin{array}{c}t+2 \\ 2\end{array}\right)$. $Z$ is said to have uniform 
position (resp. uniform position of degree $\leq k$ ) if it has uniform position in degree $t$ for all $t$ (resp. for all $t \leq k$ ). See Proposition 1 for a connection between the connectedness of the numerical character of $Z$ and the uniform position of $Z$ discovered by $\mathrm{Ph}$. Ellia and Ch. Peskine ([12]).

We work over an algebraically closed field $\mathbb{K}$. For the case of an arbitrary perfect field, see Remarks 6 and 7 .

\section{The Negative And the Positive sides}

Terminology: a lemma is either something needed later in this paper or a minor proposition.

We often silently use the following well-known lemmas.

Lemma 1. Let $Z \subset \mathbb{P}^{r}$ be a zero-dimensional scheme. Fix any subscheme $W \subseteq Z$ and any $t \in \mathbb{N}$. Then $h^{1}\left(\mathcal{I}_{W}(t)\right) \leq h^{1}\left(\mathcal{I}_{Z}(t)\right)$.

Proof. The integer $h^{1}\left(\mathcal{I}_{Z}(t)\right)$ is the dimension of the cokernel of the restriction map $H^{0}\left(\mathbb{P}^{2}, \mathcal{O}_{\mathbb{P}^{2}}(t)\right) \rightarrow H^{0}\left(Z, \mathcal{O}_{Z}(t)\right)$ (and the same is true for $W$ ). Let $\mathcal{I}_{W, Z}$ denote the ideal sheaf of $W$ in $Z$. Since $Z$ is zero-dimensional, we have $h^{1}\left(Z, \mathcal{I}_{W, Z}(t)\right)=0$. Hence the restriction map $H^{0}\left(Z, \mathcal{O}_{Z}(t)\right) \rightarrow$ $H^{0}\left(W, \mathcal{O}_{W}(t)\right)$ is surjective. Hence $h^{1}\left(\mathcal{I}_{W}(t)\right) \leq h^{1}\left(\mathcal{I}_{Z}(t)\right)$.

Lemma 2. Fix an integer $t$ and a zero-dimensional scheme $Z \subset \mathbb{P}^{r}$. We have $h^{1}\left(\mathcal{I}_{A}(t)\right)<h^{1}\left(\mathcal{I}_{Z}(t)\right)$ for all $A \subsetneq Z \Longleftrightarrow h^{1}\left(\mathcal{I}_{B}(t)\right)<h^{1}\left(\mathcal{I}_{Z}(t)\right)$ for all $B \subset Z$ such that $\operatorname{deg}(B)=\operatorname{deg}(Z)-1 \Longleftrightarrow h^{0}\left(\mathcal{I}_{B}(t)\right)=h^{0}\left(\mathcal{I}_{Z}(t)\right)$ for all $B \subset Z$ such that $\operatorname{deg}(B)=\operatorname{deg}(Z)-1$.

Proof. Fix $A \subsetneq Z$. There is a zero-dimensional scheme $B$ such that $A \subseteq B \subset$ $Z$ and $\operatorname{deg}(B)=\operatorname{deg}(Z)-1$. We have $h^{1}\left(\mathcal{I}_{A}(t)\right) \leq h^{1}\left(\mathcal{I}_{B}(t)\right)$ (Lemma 1). Hence $h^{1}\left(\mathcal{I}_{A}(t)\right)<h^{1}\left(\mathcal{I}_{Z}(t)\right)$ if $h^{1}\left(\mathcal{I}_{B}(t)\right)<h^{1}\left(\mathcal{I}_{Z}(t)\right)$. We have $h^{0}\left(\mathcal{I}_{Z}(t)\right)-$ $h^{1}\left(\mathcal{I}_{Z}(t)\right)=\left(\begin{array}{c}t+r \\ r\end{array}\right)-\operatorname{deg}(Z)$ and $h^{0}\left(\mathcal{I}_{B}(t)\right)-h^{1}\left(\mathcal{I}_{B}(t)\right)=\left(\begin{array}{c}t+r \\ r\end{array}\right)-\operatorname{deg}(B)$. Since $\operatorname{deg}(B)=\operatorname{deg}(Z)-1$, we have $h^{1}\left(\mathcal{I}_{B}(t)\right)<h^{1}\left(\mathcal{I}_{Z}(t)\right) \Longleftrightarrow h^{1}\left(\mathcal{I}_{B}(t)\right)=$ $h^{1}\left(\mathcal{I}_{Z}(t)\right)-1 \Longleftrightarrow h^{0}\left(\mathcal{I}_{B}(t)\right)=h^{0}\left(\mathcal{I}_{Z}(t)\right)$.

The next result was proved in [12], but stated only for the uniform position.

Proposition 1. Let $Z \subset \mathbb{P}^{2}$ be a zero-dimensional scheme with numerical character $n_{0}, \ldots, n_{s-1}$. If $Z$ is in uniform position in degree $\leq t$, then either $n_{0}, \ldots, n_{s-1}$ has no gap or the last gap of $n_{0}, \ldots, n_{s-1}$ is at least $t+1$.

Proof. Assume that $n_{0}, \ldots, n_{s-1}$ has gaps and call $k \in\{1, \ldots, s-1\}$ the last gap of $n_{0}, \ldots, n_{s-1}$. By [12], the proposition on page 112, there is a degree $k$ curve $T \subset \mathbb{P}^{2}$ such that the zero-dimensional scheme $E:=Z \cap T$ has numerical character $n_{0}, \ldots, n_{k-1}$. Since $k$ is a gap of $n_{0}, \ldots, n_{s-1}$, we have $n_{k} \leq n_{k-1}-2$. Since $n_{k} \geq n_{s-1} \geq s$, we have $n_{k-1} \geq s+2$. Since 
$n_{i} \geq n_{k-1}$ for all $i \leq k-1$, we have $\operatorname{deg}(E)=\sum_{i=0}^{k-1} n_{i}-k(k-1) / 2 \geq$ $k(s+2)-k(k-1) / 2=k(2 s-k+5) / 2 \geq k(k+7) / 2 \geq\left(\begin{array}{c}k+2 \\ 2\end{array}\right)$. Since $k<s$, we have $h^{0}\left(\mathcal{I}_{Z}(k)\right)=0$. Hence $E \subsetneq Z$. Since $E \subseteq Z \cap T, Z$ is not in uniform position in degree $k$. Hence $k>t$.

Proposition 2. Let $Z \subset \mathbb{P}^{2}$ be a zero-dimensional scheme. Let $n_{0}, \ldots, n_{s-1}$ be its numerical character. Hence $s(Z)=s$.

(a) We have $\tau(Z)=s-2$ if and only if $Z$ has maximal rank and $\operatorname{deg}(Z)=\left(\begin{array}{c}s+1 \\ 2\end{array}\right)$

(b) $\tau(Z)=s-1 \Longleftrightarrow Z$ has maximal rank and $\operatorname{deg}(Z) \neq\left(\begin{array}{c}s+1 \\ 2\end{array}\right) \Longleftrightarrow Z$ has maximal rank and $\left(\begin{array}{c}s+1 \\ 2\end{array}\right)<\operatorname{deg}(Z)<\left(\begin{array}{c}s+2 \\ 2\end{array}\right)$.

Proof. Since $Z \neq \emptyset$, we have $s>0$. Since $s(Z)=s$, we have $\operatorname{deg}(Z) \geq\left(\begin{array}{c}s+1 \\ 2\end{array}\right)$. Since $\tau(Z)=n_{0}-2$ and $n_{s-1} \geq s$, we have $\tau(Z)=s-2$ if and only if $n_{0}=n_{s-1}=s$, i.e. if and only if $Z$ has maximal rank and $h^{1}\left(\mathcal{I}_{Z}(s-1)\right)=$ 0 , i.e. (since $\left.h^{0}\left(\mathcal{I}_{Z}(s-1)\right)=0\right)$ if and only if $Z$ has maximal rank and $\operatorname{deg}(Z)=\left(\begin{array}{c}s+1 \\ 2\end{array}\right)$. In the same way we prove part (b).

Remark 1. Fix $t \in \mathbb{Z}$. Let $Z \subset \mathbb{P}^{r}, r \geq 2$, be a zero-dimensional scheme. We may extend verbatim the definitions of $\tau(Z), s(Z)$, maximal rank, strong defectivity, and uniform position to the case $r>2$. Assume that $Z$ has maximal rank. We have $\tau(Z)<s(Z)$. Set $s:=s(Z)$. We have $\left(\begin{array}{c}r+s-1 \\ r\end{array}\right) \leq \operatorname{deg}(Z)<\left(\begin{array}{c}r+s \\ r\end{array}\right)$. If $\operatorname{deg}(Z)>\left(\begin{array}{c}r+s-1 \\ r\end{array}\right)$, then $\tau(Z)=s-1$. If $\operatorname{deg}(Z)=\left(\begin{array}{c}r+s-1 \\ r\end{array}\right)$, then $\tau(Z)=s-2$. We may also introduce the following definition. $Z$ is said to be maximally degenerate in degree $t$ if $h^{1}\left(\mathcal{I}_{Z}(t)\right)>0$ and $h^{1}\left(\mathcal{I}_{W}(t)\right)<\operatorname{deg}(W)-\operatorname{deg}(Z)+h^{1}\left(\mathcal{I}_{Z}(t)\right)$ for every zero-dimensional scheme $W \supsetneq Z$, i.e. if and only $h^{1}\left(\mathcal{I}_{W}(t)\right)=h^{1}\left(\mathcal{I}_{Z}(t)\right)$ for every zerodimensional scheme $W \supset Z$ with $\operatorname{deg}(W)=\operatorname{deg}(Z)+1$, i.e. if and only if $h^{1}\left(\mathcal{I}_{Z}(t)\right)>0$ and $Z$ is the scheme-theoretic base locus of the linear system $\left|\mathcal{I}_{Z}(t)\right|$.

Remark 2. By (1) we have $h^{1}\left(\mathcal{I}_{Z}(x)\right)<h^{1}\left(\mathcal{I}_{Z}(y)\right)$ for all integers $x, y$ such that $\tau(Z) \geq x>y \geq s$. Hence if $h^{1}\left(\mathcal{I}_{Z}(t)\right)>0$ and $t \geq s$, then $t \geq \tau(Z)+1-h^{1}\left(\mathcal{I}_{Z}(t)\right)$. If $t<s$, then $h^{1}\left(\mathcal{I}_{Z}(t)\right)=\operatorname{deg}(Z)-\left(\begin{array}{c}t+2 \\ 2\end{array}\right)$.

Lemma 3. Fix a positive integer $t$. Let $Z \subset \mathbb{P}^{2}$ be a zero-dimensional scheme such that $s(Z) \leq t$ and $h^{1}\left(\mathcal{I}_{Z}(t)\right)>h^{1}\left(\mathcal{I}_{A}(t)\right)$ for all $A \subsetneq Z$. Fix $Z^{\prime} \subset Z$ such that $\operatorname{deg}\left(Z^{\prime}\right)=\operatorname{deg}(Z)-1$. Then $s(Z)=s\left(Z^{\prime}\right)$.

Proof. Obviously $s\left(Z^{\prime}\right) \leq s(Z)$. Assume $s\left(Z^{\prime}\right)<s(Z)$. Since $h^{1}\left(\mathcal{I}_{Z}(t)\right)>$ $h^{1}\left(\mathcal{I}_{Z^{\prime}}(t)\right)$ and $\operatorname{deg}\left(Z^{\prime}\right)=\operatorname{deg}(Z)-1$, we have $h^{0}\left(\mathcal{I}_{Z^{\prime}}(t)\right)=h^{0}\left(\mathcal{I}_{Z}(t)\right)$, i.e. $Z$ is contained in the base locus of $\left|\mathcal{I}_{Z^{\prime}}(t)\right|$. Since $t \geq s\left(Z^{\prime}\right), Z$ is contained in the base locus of $\left|\mathcal{I}_{Z^{\prime}}\left(s\left(Z^{\prime}\right)\right)\right|$. The definition of the integer $s\left(Z^{\prime}\right)$ gives $\left|\mathcal{I}_{Z^{\prime}}\left(s\left(Z^{\prime}\right)\right)\right| \neq \emptyset$. Hence $s(Z) \leq s\left(Z^{\prime}\right)$. 
Lemma 4. Let $Z \subset \mathbb{P}^{2}$ be a zero-dimensional scheme whose numerical character $n_{0}, \ldots, n_{s-1}$ is connected. Then $s n_{0}-s(s-1) \leq \operatorname{deg}(Z) \leq s n_{0}-$ $s(s-1) / 2$ and $s n_{s-1}-s(s-1) / 2 \leq \operatorname{deg}(Z) \leq s n_{s-1}$

Proof. We have $\operatorname{deg}(Z)=\sum_{i=0}^{s-1}\left(n_{i}-i\right)$. Since the numerical character of $Z$ is connected, we have $s n_{0}-s(s-1) / 2 \leq \sum_{i=0}^{s-1} n_{i} \leq s n_{0}$ and $s n_{s-1} \leq$ $\sum_{i=0}^{s-1} n_{i} \leq s n_{s-1}+s(s-1) / 2$.

Lemma 5. Let $Z \subset \mathbb{P}^{2}$ be a zero-dimensional scheme. Set $\tau:=\tau(Z)$ and call $n_{0}, \ldots, n_{s-1}$ the numerical character of $Z$. Set $e:=h^{1}\left(\mathcal{I}_{Z}(\tau)\right)$. Then $e$ is the number of integers $i \in\{0, \ldots, s-1\}$ such that $n_{i}=n_{0}$.

Proof. We have $e=\sum_{i=1}^{s-1}\left(\max \left\{0, n_{i}-\tau-1\right\}-\max \{0, i-\tau-1\}\right)$. Since $\tau=n_{0}-2$ and $n_{0} \geq n_{s-1} \geq s$, we have $i-\tau-1 \leq 0$ for all $i \leq s-1$ and $n_{i}-\tau-1>0 \Longleftrightarrow n_{i}=n_{0} \Longleftrightarrow n_{i}-\tau-1=1$.

Lemma 6. Let $Z \subset \mathbb{P}^{2}$ be a zero-dimensional scheme. Set $\tau:=\tau(Z)$. If $h^{1}\left(\mathcal{I}_{Z}(\tau)\right)>h^{1}\left(\mathcal{I}_{Z^{\prime}}(\tau)\right)$ for all $Z^{\prime} \subsetneq Z$, then the numerical character of $Z$ is connected.

Proof. Assume that the numerical character $n_{0}, \ldots, n_{s-1}$ of $Z$ is not connected and call $t \in\{1, \ldots, s-1\}$ one of its gaps, i.e. assume $n_{t} \leq n_{t-1}-2$. By [12], Proposition at page 112, there is a degree $t$ curve $T$ such that the scheme $Z_{1}:=Z \cap T$ has numerical character $n_{0}, \ldots, n_{t-1}$. Since $n_{t}<n_{t-1}$, in the strings $n_{0}, \ldots, n_{s-1}$ and $n_{0}, \ldots, n_{s-1}$ the integer $n_{0}$ appears the same number of times. Apply Lemma 5 to $Z$ and to $Z_{1}$.

Proposition 3. Let $Z \subset \mathbb{P}^{2}$ be a zero-dimensional scheme whose numerical character $n_{0}, \ldots, n_{s-1}$ is not connected. Let $m$ be the last gap of $n_{0}, \ldots, n_{s-1}$. Fix any integer $t \geq s$ such that $h^{1}\left(\mathcal{I}_{A}(t)\right)<h^{1}\left(\mathcal{I}_{Z}(t)\right)$ for all $A \subsetneq Z$. Then $t \leq n_{m}-2$.

Proof. Set $e:=h^{1}\left(\mathcal{I}_{Z}(t)\right)$. Taking $A=\emptyset$ we get $e>0$. By [12], Proposition at page 112 , there is a degree $m$ curve $T \subset \mathbb{P}^{2}$ such that the scheme $E:=$ $Z \cap T$ has numerical character $n_{0}, \ldots, n_{m-1}$. Since $m<s$, we have $E \subsetneq Z$. Hence $h^{1}\left(\mathcal{I}_{E}(t)\right)<h^{1}\left(\mathcal{I}_{Z}(t)\right)$. Formula (1) gives $n_{m}-t-1>0$, i.e. $t \leq$ $n_{m}-2$.

Remark 3. Let $A \subset \mathbb{P}^{2}$ be a zero-dimensional scheme with numerical character $n_{0}, \ldots, n_{s-1}$. Formula (1) shows that the numerical character of $A$ determines the Hilbert function $h_{A}$ of $A$. We recall how to get the numerical character of $A$ in terms of its Hilbert function. The integer $n_{0}-2$ is the maximal integer $t$ such that $h^{1}\left(\mathcal{I}_{A}(t)\right)>0$. The integer $h^{1}\left(\mathcal{I}_{A}\left(n_{0}-2\right)\right)$ is the number of the integers $i$ such that $n_{i}=n_{0}$. Fix an integer $k \in\{1, \ldots, s-1\}$ such that $n_{k}<n_{k-1}$. Assume we have proved that the values $h_{A}(t)$ for all 
integers $t \geq n_{k-1}-1$ give the string $n_{0}, \ldots, n_{k-1}$. By (1) the integer $n_{k}$ is the largest integer $t \leq n_{k-1}$ such that $h^{1}\left(\mathcal{I}_{A}(t-1)\right)-h^{1}\left(\mathcal{I}_{A}(t-2)\right)>k$.

Lemma 7. Let $Z \subset \mathbb{P}^{2}$ be a zero-dimensional scheme with numerical character $n_{0}, \ldots, n_{s-1}$.

(i) We have $h^{0}\left(\mathcal{I}_{Z}(s)\right)=1+\alpha$, where $\alpha$ is the number of integers $i$ with $n_{i}=s$.

(ii) $h^{0}\left(\mathcal{I}_{Z}(s)\right)=1$ if and only if $n_{s-1}>s$.

Proof. We have $h^{0}\left(\mathcal{I}_{Z}(s)\right)-h^{1}\left(\mathcal{I}_{Z}(s)\right)=\left(\begin{array}{c}s+2 \\ 2\end{array}\right)-\operatorname{deg}(Z)$. Since $h^{0}\left(\mathcal{I}_{Z}(s-\right.$ $1))=0$, we have $h^{1}\left(\mathcal{I}_{Z}(s-1)\right)=\left(\begin{array}{c}s+1 \\ 2\end{array}\right)-\operatorname{deg}(Z)$. Apply (1) first for $t=s$ and then for $t=s-1$. We get $h^{1}\left(\mathcal{I}_{Z}(s-1)\right)-h^{1}\left(\mathcal{I}_{Z}(s)\right)=s-\alpha$. Hence $h^{0}\left(\mathcal{I}_{Z}(s)\right)=1+\alpha$.

Part (ii) follows from part (i).

Proposition 4. Let $Z \subset \mathbb{P}^{2}$ be a zero-dimensional scheme. Set $s:=s(Z)$. We have $h^{1}\left(\mathcal{I}_{Z}(t)\right)=\operatorname{deg}(Z)-\left(\begin{array}{c}t+2 \\ 2\end{array}\right)$ for every $t \in\{0, \ldots, s-1\}$.

(i) $h^{1}\left(\mathcal{I}_{A}(s-1)\right)<h^{1}\left(\mathcal{I}_{Z}(s-1)\right)$ for each $A \subsetneq Z$ if and only if $h^{0}\left(\mathcal{I}_{B}(s-\right.$ 1)) $=0$ for each $B \subset Z$ with $\operatorname{deg}(B)=\operatorname{deg}(Z)-1$.

(ii) If $h^{0}\left(\mathcal{I}_{Z}(s)\right)=1$, then $h^{0}\left(\mathcal{I}_{A}(s-1)\right)<h^{1}\left(\mathcal{I}_{Z}(s-1)\right)$ for each $A \subsetneq Z$.

(iii) For each $t \leq s-2$ we have $h^{1}\left(\mathcal{I}_{A}(t)\right)<h^{1}\left(\mathcal{I}_{Z}(t)\right)$ for any $A \subsetneq Z$.

(iv) Fix an integer $t<s . Z$ is strongly defective in degree $t$ if and only if $\gamma:=\operatorname{deg}(Z)-\left(\begin{array}{c}t+2 \\ 2\end{array}\right)>0$ and $\operatorname{deg}(Z \cap T)<\operatorname{deg}(Z)-\gamma$ for each $T \in\left|\mathcal{O}_{\mathbb{P}^{2}}(t)\right|$. If $t \leq s-2$, then $\gamma \geq\left(\begin{array}{c}s+1 \\ 2\end{array}\right)-\left(\begin{array}{c}t+2 \\ 2\end{array}\right)>0$.

Proof. Let $\mathcal{B}$ denote the set of all zero-dimensional schemes $B \subset Z$ such that $\operatorname{deg}(B)=\operatorname{deg}(Z)-1$. Fix any integer $t \leq s-1$. If there is $A \subsetneq Z$ such that $h^{1}\left(\mathcal{I}_{A}(t)\right)=h^{1}\left(\mathcal{I}_{Z}(t)\right)$, then $h^{1}\left(\mathcal{I}_{B}(t)\right)=h^{1}\left(\mathcal{I}_{B}(t)\right)=h^{1}\left(\mathcal{I}_{Z}(t)\right)$ for some $B \in \mathcal{B}$ (Lemma 2). For any $B \in \mathcal{B}$ we have $h^{0}\left(\mathcal{I}_{B}(t)\right)=h^{0}\left(\mathcal{I}_{Z}(t)\right)=$ 0 (Lemma 2 and the inequality $t<s$ ). If $B \in \mathcal{B}$ and $h^{0}\left(\mathcal{I}_{B}(t)\right)=0$, then $h^{1}\left(\mathcal{I}_{B}(t)\right)=\operatorname{deg}(B)-\left(\begin{array}{c}t+2 \\ 2\end{array}\right)=h^{1}\left(\mathcal{I}_{Z}(t)\right)-1$. Now assume $t \leq s-$ 2. If $h^{0}\left(\mathcal{I}_{B}(t)\right)>0$, then $h^{0}\left(\mathcal{I}_{B}(s-1)\right) \geq 3$ and hence $h^{0}\left(\mathcal{I}_{Z}(s-1)\right) \geq$ 2 , a contradiction. If $h^{0}\left(\mathcal{I}_{B}(s-1)\right)>0$, then $h^{0}\left(\mathcal{I}_{B}(s)\right) \geq 3$ and hence $h^{0}\left(\mathcal{I}_{Z}(s)\right) \geq 2$. We just proved parts (i), (ii), and (iii).

Now we prove part (iv). Since $\operatorname{deg}(Z) \geq\left(\begin{array}{c}s+1 \\ 2\end{array}\right)$, we have $\gamma \geq\left(\begin{array}{c}s+1 \\ 2\end{array}\right)-\left(\begin{array}{c}t+2 \\ 2\end{array}\right)$. Hence $\gamma>0$ if $t \leq s-2$. Fix $A \subsetneq Z$. First assume $w:=\operatorname{deg}(Z)-\operatorname{deg}(A) \leq \gamma$. Since $h^{0}\left(\mathcal{I}_{A}(t)\right)-h^{1}\left(\mathcal{I}_{A}(t)\right)=\left(\begin{array}{c}t+2 \\ 2\end{array}\right)-\operatorname{deg}(A)$, we have $h^{1}\left(\mathcal{I}_{A}(t)\right)=\gamma-w$ if and only if $h^{0}\left(\mathcal{I}_{A}(w)\right)=0$. Now assume $w>\gamma$. There is a zero-dimensional scheme $A_{1}$ such that $A \subset A_{1} \subset Z$ and $\operatorname{deg}\left(A_{1}\right)=\operatorname{deg}(Z)-\gamma$. We have $h^{1}\left(\mathcal{I}_{A}(t)\right) \leq h^{1}\left(\mathcal{I}_{A_{1}}(t)\right)$ (Lemma 1$)$. We just proved that $h^{1}\left(\mathcal{I}_{A_{1}}(t)\right)=0$ if and only if $h^{0}\left(\mathcal{I}_{A_{1}}(t)\right)=0$, concluding the proof of part (iv). 
Proposition 5. Let $E \subset \mathbb{P}^{2}$ be a zero-dimensional scheme, $E \neq \emptyset$. Let $n_{0}, \ldots, n_{s-1}$ be the numerical character of E. Fix an integer $d$ such that $s-2 \leq d \leq n_{s-1}-2$. Set $\epsilon:=h^{1}\left(\mathcal{I}_{E}(d)\right)$. Then $h^{1}\left(\mathcal{I}_{A}(d)\right)<\epsilon$ for all $A \subsetneq E$ (even if $A=\emptyset$ )

Proof. The lemma is true for $A=\emptyset$, because (1) gives $\epsilon>0$ (here we use the inequalities $s-1-d-1 \leq 0$ and $\left.n_{s-1}-d-1>0\right)$. Now assume $A \neq \emptyset$ and call $m_{0}, \ldots, m_{r-1}$ the numerical character of $A$. Obviously $r \leq s$. Let $t$ be the maximal integer $\leq s-1$ such that $m_{t} \geq d+2$ with the convention $t=-1$ if there is no such integer. If $t=-1$, then (1) gives $h^{1}\left(\mathcal{I}_{A}(d)\right)=0$. Hence we may assume $t \in\{0, \ldots, r-1\}$. Use (1) for $Z$ and $A$ with $t:=d$. Since $d \geq s-2$, we have $\max \{0, s-1-d-1\}=0$ and $\max \{0, r-1-d-1\}=0$. Hence $\epsilon=\sum_{i=0}^{s-1}\left(n_{i}-d-1\right)=\sum_{i=0}^{t}\left(n_{i}-d-1\right)+\sum_{i=t+1}^{s-1}\left(n_{i}-d-1\right)$ and $h^{1}\left(\mathcal{I}_{A}(d)\right)=\sum_{i=0}^{t}\left(m_{i}-d-1\right)$. Hence $h^{1}\left(\mathcal{I}_{A}(d)\right)=\epsilon$ if and only if $\sum_{i=0}^{t} m_{i}=\sum_{i=0}^{s-1} n_{i}$ (or, by Lemma 1 , if and only if $\sum_{i=0}^{t} m_{i} \geq \sum_{i=0}^{s-1} n_{i}$ ). We have $\operatorname{deg}(E)=\sum_{i=0}^{s-1}\left(n_{i}-i\right)=\sum_{i=0}^{t}\left(n_{i}-i\right)+\sum_{i=t+1}^{s-1}\left(n_{i}-i\right)$ and $\operatorname{deg}(A)=\sum_{i=0}^{r-1}\left(m_{i}-i\right)=\sum_{i=0}^{t}\left(m_{i}-i\right)+\sum_{i=t+1}^{r-1}\left(m_{i}-i\right)$. Since $m_{j}>j$ for all $j<r$ and $n_{h}>h$ for all $h<s$, we have $\sum_{i=0}^{t} m_{i} \geq \sum_{i=0}^{k-1} n_{i}$ if and only if $t=r-1=s-1$ and $\operatorname{deg}(A)=\operatorname{deg}(E)$, i.e. if and only if $A=E$, a contradiction.

If $d \in\{s-2, s-1\}$, then Proposition 5 is a particular case of Proposition 4 .

The following examples shows that Proposition 5 cannot be improved, without making some assumptions not involving only the numerical character.

Example 1. Fix a zero-dimensional scheme $W \subset \mathbb{P}^{2}$ with numerical character $a_{0}, \ldots, a_{s-1}$ such that $a_{s-1}=s$, i.e. such that $b:=h^{0}\left(\mathcal{I}_{W}(s)\right) \geq 2$ (Lemma 7). Take a general $P \in \mathbb{P}^{2}$. Set $Z:=W \cup\{P\}$. Since $P$ is general, we have $h^{0}\left(\mathcal{I}_{Z}(t)\right)=h^{0}\left(\mathcal{I}_{W}(t)\right)-1$ for all $t \geq s$. Hence $h^{1}\left(\mathcal{I}_{Z}(t)\right)=h^{1}\left(\mathcal{I}_{W}(t)\right)$ for all $t \geq s$. Since $b \geq 2$, we have $s(Z)=s$. We get that the numerical character $n_{0}, \ldots, n_{s-1}$ of $Z$ satisfies $n_{s-b+1}=a_{s-b+1}+1=s+1$ and $n_{i}=a_{i}$ for all $i \neq s-b+1$.

Example 2. Fix a zero-dimensional scheme $W \subset \mathbb{P}^{2}$ with numerical character $a_{0}, \ldots, a_{s-1}$ such that $a_{s-1}>s$, i.e. such that $h^{0}\left(\mathcal{I}_{W}(s)\right)=1$ (Lemma 7). Take a general $P \in \mathbb{P}^{2}$. Set $Z:=W \cup\{P\}$. Since $P$ is general, we have $h^{0}\left(\mathcal{I}_{Z}(t)\right)=h^{0}\left(\mathcal{I}_{W}(t)\right)-1$ for all $t \geq s$. Hence $h^{1}\left(\mathcal{I}_{Z}(t)\right)=h^{1}\left(\mathcal{I}_{W}(t)\right)$ for all $t \geq s$ and $s(Z)=s+1$. Let $n_{0}, \ldots, n_{s+1}$ be the numerical character of $Z$. We have $n_{i}=a_{i}$ for all $i \leq s-1$ and $n_{s}=s+1$.

Proposition 6. Let $Z \subset \mathbb{P}^{2}$ be a zero-dimensional scheme with numerical character $n_{0}, \ldots, n_{s-1}$. Assume the existence of an integer $t>0$ such that $e:=h^{1}\left(\mathcal{I}_{Z}(t)\right)>0$. Then we have $t \leq n_{0}-2$. 
(a) Assume that $n_{0}, \ldots, n_{s-1}$ is not connected and call $k$ its last gap. Assume $h^{1}\left(\mathcal{I}_{A}(t)\right)<e$ for all $A \subsetneq Z$. Then $t \leq n_{k}-2$. If $t \geq s$, then $e=\sum_{i=0}^{s-1} \max \left\{0, n_{i}-t-1\right\}$.

(b) Assume that $n_{0}, \ldots, n_{s-1}$ is connected. If $s \geq n_{0}-t$, then $e \geq$ $\left(n_{0}-t-1\right)\left(n_{0}-t\right) / 2$.

Proof. Since $\tau(Z)=n_{0}-2$, we have $t \leq n_{0}-2$.

(i) Take the set-up of part (a). Assume that $n_{0}, \ldots, n_{s-1}$ is not connected and call $k$ its last gap. There is a degree $k$ curve $T \subset \mathbb{P}^{2}$ such that $E:=Z \cap T$ has numerical character $n_{0}, \ldots, n_{k-1}$. By (1) applied first to $Z$ and then to $E$ we get that $h^{1}\left(\mathcal{I}_{E}(y)\right)=h^{1}\left(\mathcal{I}_{Z}(y)\right)$ if and only if $y \geq n_{k}-1$. The value for $e$ is given by $(1)$.

(ii) Assume that $n_{0}, \ldots, n_{s-1}$ is connected. Hence $n_{i} \geq n_{0}-i$ for all $i$. If $s \geq n_{0}-t$, then $(1)$ gives $e \geq \sum_{i=0}^{n_{0}-t-2}\left(n_{i}-t-1\right) \geq \sum_{i=0}^{n_{0}-t-2}\left(n_{0}-t-1-i\right)=$ $\left(n_{0}-t-1\right)\left(n_{0}-t\right) / 2$.

\section{THE DECOMPOSITION FOR NUMERICAL CHARACTERS WITH GAPS}

In this paper a curve $T \subset \mathbb{P}^{2}$ is an effective divisor, i.e. it has no embedded point.

For any curve $T \subset \mathbb{P}^{2}$ and any zero-dimensional scheme $Z \subset \mathbb{P}^{2}$ the residual scheme $\operatorname{Res}_{T}(Z)$ of $Z$ with respect to $T$ is the closed subscheme of $\mathbb{P}^{2}$ with $\mathcal{I}_{T}: \mathcal{I}_{Z}$ as its ideal sheaf. Set $k:=\operatorname{deg}(T)$. For any $y \in \mathbb{Z}$ we have an exact sequence

$$
0 \rightarrow \mathcal{I}_{\operatorname{Res}_{T}(Z)}(y-k) \rightarrow \mathcal{I}_{Z}(y) \rightarrow \mathcal{I}_{Z \cap T, T}(y) \rightarrow 0
$$

Notice that $\operatorname{Res}_{T}(Z) \subseteq Z$. Taking the Hilbert polynomials in (2) we get $\operatorname{deg}(Z)=\operatorname{deg}(Z \cap T)+\operatorname{deg}\left(\operatorname{Res}_{T}(Z)\right)$. If $H \subset \mathbb{P}^{2}$ is any curve, then $\operatorname{Res}_{H}\left(\operatorname{Res}_{T}(Z)\right)=\operatorname{Res}_{T+H}(Z)=\operatorname{Res}_{T}\left(\operatorname{Res}_{H}(Z)\right)$, in which $T+H$ is the sum as divisors, i.e. counting the multiplicities of the irreducible components.

For any integer $d>0$ set $N(d):=d(d+3) / 2$. Let $\nu_{d}: \mathbb{P}^{2} \rightarrow \mathbb{P}^{N(d)}$ denote the order $d$ Veronese embedding of $\mathbb{P}^{2}$, i.e. the embedding of $\mathbb{P}^{2}$ induced by the complete linear system $\left|\mathcal{O}_{\mathbb{P}^{2}}(d)\right|$. For any zero-dimensional scheme $W \subset \mathbb{P}^{N(d)}$ let $\langle W\rangle$ denote the linear span of $W$, i.e. the intersection of all hyperplanes containing $W$, with the convention $\langle W\rangle=\mathbb{P}^{N(d)}$ if there is no hyperplane. Notice that $\langle\emptyset\rangle=\emptyset$.

Lemma 8. Let $Z \subset \mathbb{P}^{2}$ be a zero-dimensional scheme whose numerical character $n_{0}, \ldots, n_{s-1}$ is not connected. Let $k$ be any gap of the numerical character $n_{0}, \ldots, n_{s-1}$. There is a unique degree $k$ effective divisor $T \subset \mathbb{P}^{2}$ such that $E:=Z \cap T$ has numerical character $n_{0}, \ldots, n_{k-1}$. Moreover, the scheme $\operatorname{Res}_{T}(Z)$ has numerical character $m_{0}, \ldots, m_{s-k-1}$, where $m_{i}:=n_{i}-$ $k$ for all $i$. 
Proof. The existence of $T$ and the "Moreover" part are true by [12], the proposition on page 112. Set $d:=n_{k-1}-2$ and $\epsilon:=h^{1}\left(\mathcal{I}_{Z}(d)\right)$. Assume that $H \subset \mathbb{P}^{2}$ is another degree $k$ effective divisor such that $F:=Z \cap H$ has numerical character $n_{0}, \ldots, n_{k-1}$. Since $n_{0}, \ldots, n_{k-1}$ is the numerical character of $E$ and $F, n_{0}, \ldots, n_{s-1}$ is the numerical character of $Z$ and $n_{k} \leq n_{k-1}-2=d$, (1) gives $h^{1}\left(\mathcal{I}_{E}(d)\right)=h^{1}\left(\mathcal{I}_{F}(d)\right)=\epsilon$. Since $E \subseteq E \cup F \subseteq Z$, Lemma 1 gives $h^{1}\left(\mathcal{I}_{E}(d)\right) \leq h^{1}\left(\mathcal{I}_{E \cup F}(d)\right) \leq h^{1}\left(\mathcal{I}_{Z}(d)\right)$. Hence $h^{1}\left(\mathcal{I}_{E \cup F}(d)\right)=\epsilon$. The definition of the Veronese embedding $\nu_{d}$ gives $\operatorname{dim}\left(\left\langle\nu_{d}(E)\right\rangle\right)=\operatorname{deg}(E)-1-\epsilon$, $\operatorname{dim}\left(\left\langle\nu_{d}(F)\right\rangle\right)=\operatorname{deg}(F)-1-\epsilon$ and $\operatorname{dim}\left(\left\langle\nu_{d}(E \cup F)\right\rangle\right)=\operatorname{deg}(E)-1-\epsilon$. Since $E \cap F \subseteq E$, Lemma 1 gives $\operatorname{dim}\left(\left\langle\nu_{d}(E \cap F)\right\rangle \geq \operatorname{deg}(E \cap F)-1-\epsilon\right.$. Since $\operatorname{deg}(E)+\operatorname{deg}(F)=\operatorname{deg}(E \cup F)+\operatorname{deg}(E \cap F)$, Grassmann's formula gives $\left.\operatorname{dim}\left(\left\langle\nu_{d}(E)\right\rangle\right) \cap\left\langle\nu_{d}(F)\right\rangle\right)=\operatorname{deg}(E \cap F)-\epsilon$. Since $\nu_{d}(E \cap F) \subset$ $\left\langle\nu_{d}(E)\right\rangle \cap\left\langle\nu_{d}(F)\right\rangle$, we get $\operatorname{dim}\left(\left\langle\nu_{d}(E \cap F)\right\rangle=\operatorname{deg}(E \cap F)-1-\epsilon\right.$, i.e. $h^{1}\left(\mathcal{I}_{E \cap F}(d)\right)=\epsilon$. Lemma 5 gives $E \cap F=E$, i.e. $E=F$, a contradiction.

Remark 4. Let $Z \subset \mathbb{P}^{2}$ be a zero-dimensional scheme whose numerical character is not connected, say it has $c$ gaps $k_{1}, \ldots, k_{c}$. Let $T_{1}, \ldots, T_{c}$, $E_{1}, \ldots, E_{c+1}, Z_{1}, \ldots, Z_{c}=E_{c+1}$ be the splitting sequence of $Z$. Set $k_{0}:=0$. We have $h^{0}\left(\mathcal{I}_{E_{i}}\left(k_{i}-k_{i-1}\right)\right)=1$ for all $i$ (Lemma 7 applied to $\left.E_{i}\right)$. Hence $T_{i}$ is the only element of $\left|\mathcal{I}_{E_{i}}\left(k_{i}-k_{i-1}\right)\right|$. For any zero-dimensional scheme $A \subset \mathbb{P}^{2}$ there is at most one integer $t$ such that $h^{0}\left(\mathcal{I}_{A}(t)\right)=1$. Hence the sequence $E_{1}, \ldots, E_{c}$ uniquely determines the curves $T_{1}, \ldots, T_{c}$ and the gaps $k_{1}, \ldots, k_{c}$. The Hilbert function of each $E_{i}$ gives its numerical character. Hence the sequence $E_{1}, \ldots, E_{c+1}$ uniquely determines the numerical character of $Z$.

Remark 5. Assume that $Z$ is reduced and that its numerical character is not connected. Let $T_{1}, \ldots, T_{c}, E_{1}, \ldots, E_{c+1}, Z_{1}, \ldots, Z_{c}=E_{c+1}$ be the splitting sequence of $Z$. In this case $E_{i} \cap E_{j}=\emptyset$ for all $i \neq j, Z=E_{1} \sqcup$ $\cdots \sqcup E_{c+1}$ and $Z_{i}=\sqcup_{h=i+1}^{c+1} E_{h}$ for all $i \in\{1, \ldots, c\}$. Hence in this case the sequence $E_{1}, \ldots, E_{c+1}$ gives $Z$ and (by Remark 4) all the data of the splitting sequence of $Z$.

Proposition 7. Let $Z \subset \mathbb{P}^{2}$ be a zero-dimensional scheme whose numerical character $n_{0}, \ldots, n_{s-1}$ is not connected. Let $k_{1}, \ldots, k_{c}$ be the gaps of $n_{0}, \ldots, n_{s-1}$ and $E_{1}, \ldots, E_{c+1}$ the splitting sequence of $Z$. Set $k_{0}:=0$. Then $h^{0}\left(\mathcal{I}_{E_{1}}\left(k_{i}-k_{i-1}\right)\right)=1$ for all $i \in\{1, \ldots, c\}$ and $h^{0}\left(\mathcal{I}_{E_{c+1}}\left(s-k_{c}\right)\right)=$ $h^{0}\left(\mathcal{I}_{Z}(s)\right)$.

Proof. We proved in Remark 4 that $h^{0}\left(\mathcal{I}_{E_{1}}\left(k_{i}-k_{i-1}\right)\right)=1$ for all $i \in$ $\{1, \ldots, c\}$. Since $E_{c+1}$ has a numerical character with length $s-k_{c}$, we have $\left|\mathcal{I}_{E_{c+1}}\left(s-k_{c}\right)\right| \neq \emptyset$. Obviously for each $A \in\left|\mathcal{I}_{E_{c+1}}\left(s-k_{c}\right)\right|$ we have $A \cup T_{1} \cup \cdots \cup T_{c} \in\left|\mathcal{I}_{Z}(s)\right|$. Let $\alpha$ be the number of integers $i \in\{0, \ldots, s-1\}$ 
such that $n_{i}=s$. We have $h^{0}\left(\mathcal{I}_{Z}(s)\right)=1+\alpha$ (Lemma 7). Recall that $E_{c+1}$ has numerical character $b_{0}, \ldots, b_{r-1}$ with $r=s-k_{c}$ and $b_{i}=n_{i+k_{c}}-k_{c}$ for all $i$ ([12], Proposition at page 112). Hence $b_{i}=s-k_{c}$ for exactly $\alpha$ indices $i$. Hence Lemma 7 gives $h^{0}\left(\mathcal{I}_{E_{c+1}}\left(s-k_{c}\right)\right)=1+\alpha=h^{0}\left(\mathcal{I}_{Z}(s)\right)$.

The next example shows that even if each term $E_{1}, \ldots, E_{c+1}$ of a splitting is reduced, then $Z$ may be unreduced and different schemes may have the same splitting sequence $E_{1}, \ldots, E_{c+1}$. Of course, the sets $E_{1}, \ldots, E_{c+1}$ cannot be pairwise disjoints if $Z$ is not reduced. In the next example we have $c=s$.

Example 3. Fix an integer $s \geq 2$ and integers $n_{0}, \ldots, n_{s-1}$ such that $n_{s-1} \geq$ $s$ and $n_{i} \leq n_{i-1}-2$ for all $i \in\{1, \ldots, s-1\}$. Fix a line $L \subset \mathbb{P}^{2}$ and sets $E_{1}, \ldots, E_{c+1}$ such that $E_{c+1} \subset \cdots \subset E_{1} \subset L$ and $\sharp\left(E_{i}\right)=n_{i-1}+i-1$ for all $i$. For each $P \in E_{1}$ fix any line $L_{P} \subset \mathbb{P}^{2}$ such that $P \in L_{P}$ and $L_{P} \neq L$. Set $Z:=\sqcup_{P \in E_{1}} Z_{P}$, where $Z_{P}$ is the degree $i$ divisor of $L_{P}$ with $P$ as its reduction and $i$ is the maximal integer with $P \in E_{i}$. In this case as splitting curves we use $s$ times the line $L$.

Remark 6. Fix an algebraically closed field $\mathbb{L} \supset \mathbb{K}$. Let $Z \subset \mathbb{P}^{2}$ be a zero-dimensional scheme defined over $\mathbb{K}$. Obviously $Z$ is defined over $\mathbb{L}$. Since the inclusion $\mathbb{K} \subset \mathbb{L}$ is flat and cohomology commutes with flat base change ([14], Proposition III.9.3), the integers $h^{1}\left(\mathcal{I}_{Z}(t)\right), t \in \mathbb{Z}$, are the same if we see $Z$ defined over $\mathbb{K}$ or over $\mathbb{L}$. Hence the Hilbert function and the numerical character of $Z$ are the same over $\mathbb{K}$ and over $\mathbb{L}$.

Remark 7. Assume that $\mathbb{K}$ is the algebraic closure of the perfect field $K$. Fix a zero-dimensional scheme $Z \subset \mathbb{P}^{2}$ defined over $K$ (we do not assume that each point of $Z_{\text {red }}$ is defined over $K$ ). Assume that the numerical character of $Z$ is not connected and call $T_{1}, \ldots T_{c}, E_{1}, \ldots, E_{c+1}, Z_{1}, \ldots, Z_{c}=$ $E_{c+1}$ the splitting sequence of $Z$. There is a finite extension $F$ of $K$ in which the constructions in [12] are defined. Hence the splitting sequence of $Z$ is defined over $L$. The uniqueness of $T_{1}, \ldots, T_{c}$ (Lemma 7 ) implies that each $T_{i}$ is fixed by $G$. Hence each $T_{i}$ is defined over $K$. Since $Z$ and $T_{1}$ are defined over $K, E_{1}:=Z \cap T_{1}$ and $Z_{1}:=\operatorname{Res}_{T_{1}}(Z)$ are defined over $K$. In $c$ steps we get that the splitting sequence of $Z$ is defined over $K$.

\section{How to USE NUMERICAL CHARACTERS TO FIND PROPERTIES OF CODES FROM PLANE CURVES} it.

How to use [12] if a reasonable example is suggested and you want to test

Let $C \subset \mathbb{P}^{2}$ be a smooth plane curve. Set $d:=\operatorname{deg}(C)$. Fix an integer $t>0$, a zero-dimensional scheme $E \subset C$ and a finite set $B \subset C \backslash E_{\text {red }}$. 
Let $\mathcal{C}_{1}$ be the affine code obtained evaluating $H^{0}\left(\mathbb{P}^{2}, \mathcal{I}_{E}(t)\right)$ at the points of $B$ (since $B$ is finite we may fix the homogeneous coordinates of each point of $B$ to evaluate each polynomial $f \in H^{0}\left(\mathbb{P}^{2}, \mathcal{I}_{E}(t)\right)$; two different choices give isometric codes). Let $\mathcal{C}$ be the code obtained evaluating at the points of $B$ the restriction to $C$ of $H^{0}\left(\mathbb{P}^{2}, \mathcal{I}_{E}(t)\right.$ ) (it can be seen as a Goppa code). If $t<d$, then the restriction map is injective and hence $\mathcal{C}^{\perp}=\mathcal{C}_{1}^{\perp}$. Anyway, $\mathcal{C}_{1}=\mathcal{C}$, even when $t \geq d$ just because $B \cup E \subset C$ and hence the evaluation map factors through the restriction to $C$. Therefore, for every $t$ we have $h^{1}\left(\mathcal{I}_{F \cup E}(t)\right)=h^{1}\left(C, \mathcal{O}_{C}(t)(-F-E)\right)$ for each $F \subseteq B$. We always assume $h^{0}\left(C, \mathcal{O}_{C}(t)(-B-E)\right)=0$, so that $\mathcal{C}$ is an $[n, k]$-code with $n:=\sharp(B)$ and $k:=h^{0}\left(C, \mathcal{O}_{C}(t)(-E)\right)$. Hence $\mathcal{C}^{\perp}$ is an $[n, n-k]$-code. The minimum distance of the code $\mathcal{C}^{\perp}$ is the minimal cardinality of a set $F \subseteq B$ such that $h^{1}\left(\mathcal{I}_{F \cup E}(t)\right)>0$. We could look at the intersections of $B \cup E$ first with lines and then with conics (reducible or smooth) and see in which range they touch our problem, i.e. if there is a line (resp. a conic) $D$ with $\operatorname{deg}(D \cap(B \cup E)) \geq t+2$ (resp. $\operatorname{deg}(D \cap(B \cup E)) \geq 2 t+2)$. Lines (resp. conics) correspond to a scheme $B^{\prime} \cup E$ with $s\left(B^{\prime} \cup E\right)=1$ (resp. $s\left(B^{\prime} \cup E\right)=2$ ) and the key step is to show that either $s(S \cup E)=1$ (resp. $s(S \cup E)=2$ ) or 1 (resp. 2) is a gap of the numerical character of $S \cup E$ for a suitable $S \subseteq B$; in both cases the minimality of $B^{\prime}$ implies $B=B^{\prime} \cap D$ with $\operatorname{deg}(D)=1$ (resp. $\operatorname{deg}(D)=2$ ).

In the case of the Hermitian curve the minimum distances where previously known for all one-point codes and two-points codes. So the main business in [6], [7] and [8] was to show that only lines or conics could give minimum distance codewords (or codewords with small weight) and then count in a certain ranges the lines with large intersection with $B$ (well-known and classical) and then the smooth conics ([11]). As far as we know there is no list of the large integers arising as $\sharp\left(C\left(\mathbb{F}_{q^{2}}\right) \cap T\right)$ with $C$ the Hermitian curve over $\mathbb{F}_{q^{2}}$ and $T$ an irreducible cubic. Hence we were forced to work only in certain ranges. For arbitrary data $C, E, B$ the paper [12] may be a first check: look for lines or conics or cubics $T$ with large $\operatorname{deg}(T \cap(E \cup B))$. The paper [12] may also help in the search of a zero-dimensional scheme $E \subset C$ with good properties. In [6], [7] and [8] there are either $P \in C \backslash B$ and an integer $a>0$ or $P, Q \in C \backslash B$ and integers $a>0, b>0$ such that either $E=a P$ or $E=a P+b Q$ (respectively one-point codes and two-points codes). One needs to find useful integers $a>0$ or $a>0, b>0$; for $a P$ (resp $a P+b Q)$ we are looking at the Weierstrass semigroup of $P$ (resp. of the pair $P, Q$ ), or at least at their first few non-gaps; in the case of the Hermitian curve these semigroups were known in advance, but in the general case [12] may be useful to compute them in certain intervals (at least as large as the ones for which one can find a useful set $B$ ). Sometimes this heuristic 
strategy helps even if the curve $C$ we are interested in has a singular plane model, but for a restricted set of integers $t([5])$.

Now we fix an integer $e \geq 2$ and look at the minimal cardinality of a set $B_{1} \subseteq B$ such that $h^{1}\left(\mathcal{I}_{B_{1} \cup E}(t)\right)=e$. This cardinality is the generalized Hamming $e$-weight of $\mathcal{C}^{\perp}$. We wrote in [1], Theorems 1 and 2, an axiomatic treatment of this subject for lines and conics in terms of the possible intersection of $B \cup E$ with lines and conics (it corresponds only to [12], Remarques at page 116). It is straightforward to go on with cubics, quartics and so on using [12], Corollaire 2, but to do this in full one would also need a reasonable application.

\section{REFERENCES}

[1] E. Ballico, Generalized Hamming weights of codes obtained from smooth plane curves, Afr. Mat., 24 (4) (2013), 565-569.

[2] E. Ballico, Finite subsets of projective spaces with bad postulation in a fixed degree, Beitr. Algebra Geom., 54 (2013), 81-103.

[3] E. Ballico and A. Bernardi, Stratification of the fourth secant variety of Veronese variety via the symmetric rank, Adv. Pure Appl. Math., 4 (2) (2013), 215-250; DOI: 10.1515/apam-2013-0015

[4] E. Ballico and L. Chiantini, A criterion for detecting the identifiability of symmetric tensors of size three, Differ. Geom. Appl., 30 (2012), 233-237.

[5] E. Ballico and A. Ravagnani, On the duals of geometric Goppa codes from norm-trace curves, Finite Fields Appl., 20 (2013), 30-39.

[6] E. Ballico and A. Ravagnani, The dual geometry of Hermitian two-point codes, Discrete Math., 313 (2013), 2687-2695.

[7] E. Ballico and A. Ravagnani, On the dual minimum distance and minimum weight of codes from a quotient of the Hermitian curve, Appl. Algebra Eng. Comm. Comput., 24 (2013), 343-354, DOI 10.1007/s00200-013-0206-z.

[8] E. Ballico and A. Ravagnani, On the geometry of Hermitian one-point codes, J. Algebra, 397 (2014), 499-514.

[9] A. Couvreur, The dual minimum distance of arbitrary dimensional algebraicgeometric codes, J. Algebra, 350 (1) (2012), 84-107.

[10] E. D. Davis, 0-dimensional subschemes of $\mathbf{P}^{2}$ : new application of Castelnuovo's function, Ann. Univ. Ferrara Sez., VII (N.S.) 32 (1986), 93-107 (1987).

[11] G. Donati and N. Duranti, On the intersection of a Hermitian curve with a conic, Des. Codes Cryptography, (2010) 57:347360, DOI 10.1007/s10623-010-9371-2.

[12] Ph. Ellia and Ch. Peskine, Groupes de points de $\mathbf{P}^{2}$ : caractère et position uniforme, Algebraic geometry (L' Aquila, 1988), 111-116, Lecture Notes in Math., 1417, Springer, Berlin, 1990.

[13] G. M. Hana and T. Johnsen, Scroll codes, Des. Codes Cryptography, 45 (3) (2007), $365-377$.

[14] R. Hartshorne, Algebraic Geometry, Springer, 1977.

[15] L. Gruson and Ch. Peskine, Genre des courbes de l'espace projectif, in Algebraic geometry (Proc. Sympos., Univ. Tromsø, Tromsø, 1977), pp. 31-59, Lecture Notes in Math., 687, Springer, Berlin, 1978. 
[16] J. M. Landsberg, Tensors: Geometry and Applications, Graduate Studies in Mathematics, Vol. 128, Amer. Math. Soc. Providence, 2012.

(Received: October 12, 2013)

Department of Mathematics

(Revised: March 8, 2014)

\author{
University of Trento \\ 38123 Povo (TN) \\ Italy \\ ballico@science.unitn.it
}

